Proceedings of the International School and Conference on Optics and Optical Materials, ISCOM07, Belgrade, Serbia, September 3-7, 2007

\title{
Optical and Magnetooptical Properties of Clinical Dextrans
}

\author{
M. Koralewski ${ }^{a, *}$, F. Reinholz ${ }^{a}$, J. Karoń $^{b}$ \\ AND K. WARACZEWSKI ${ }^{b}$ \\ ${ }^{a}$ Institute of Physics, Adam Mickiewicz University \\ Umultowska 85, 61-614 Poznań, Poland \\ ${ }^{b}$ Clinic of General Surgery, University of Medical Sciences \\ Poznań, Poland
}

\begin{abstract}
The effect of dextran concentration, $\mathrm{pH}$, temperature, and urea on the conformation of dextran chain in saline solution were studied. Two characteristic concentrations for both dextrans were observed. One with $c_{1} \approx$ $0.02 \mathrm{~g} / \mathrm{dL}$ and second with $c_{2} \sim 2 \mathrm{~g} / \mathrm{dL}$ which may be related with aggregation and overlapping processes of polymer chains, respectively. The mean optical polarizability for dextran polysaccharides were discussed in relation to their radius of gyration. Preliminary results of the Faraday effect dispersion and their temperature dependence is also presented.
\end{abstract}

PACS numbers: 78.20.Ek, 78.20.Ls, 81.05.Lg

\section{Introduction}

Dextran is a bacterial polysaccharides polymer composed of $\alpha$-Dglucopyranosyl residues, all having the $\alpha(1 \rightarrow 6)$ linkage with mainly $(1 \rightarrow 3)$ branching possible. Dextran as polymer of great significance still gains developing interest because of its possible applications in the nanoscience and nanotechnology. Dextran saline solutions as the plasma volume expander and for other purposes are commonly used in clinical practice - such polymers are called clinical dextrans [1]. Iron dextran as pharmaceutical analog of ferritin is applied for treatment of anemic iron deficiency [1]. Recently dextran based hydrogels have received increased attention due to their potential application as control delivery and release of bioactive agents [2]. Dextran and its derivatives are used as polymer coating of magnetic nanoparticles, and their application in biomedicine became very attractive [3]. Very recently use of iron dextran for construction of nanobatteries was discussed [4]. It is well known that chiroptical spectroscopies provided a sensitive probe of conformational changes of molecules, polymers, and complexes. Chiroptical properties of dextran polymer were studied by vacuum ultraviolet cir-

${ }^{*}$ corresponding author; e-mail: koral@amu.edu.pl 
cular dichroism [5], although prior to this work optical rotation mainly for yellow $D$ line was used to characterize this polysaccharide [6]. Preliminary results of optical rotatory dispersion (ORD) and the light refractive index dispersion have been published recently by authors [7]. Faraday effect dispersion i.e. magnetic optical rotatory dispersion (MORD) may be especially useful in description of metal center with polymer as ligands but this study is scarcely noticed in the literature of polysaccharides. Systematic concentration, temperature, and $\mathrm{pH}$ dependence of ORD, MORD, refractive index and optical absorption of dextrans and their metal complexes to best of our knowledge are not discussed.

Increasing interest in dextran polymers studies stimulate us to perform its optical research. It was thought that systematic study of ORD, MORD, and optical polarizability might provide additional information for better understanding of the physicochemical properties of dextran polymer and his metal complexes.

\section{Experimental}

The measurements were performed for clinical dextran $40000\left(10 \% ; M_{\mathrm{w}}=\right.$ $40,000-$ Dex40) and $70000\left(6 \% ; M_{\mathrm{w}}=70,000-\right.$ Dex70) aqueous saline solution obtained from Fresenius Kabi or Baxter. Different aqueous solutions with saline $(0.9 \% \mathrm{NaCl}$ - Fresenius Kabi) used as a solvent were obtained by dilution of the stock solutions. The $\mathrm{pH}$ of solutions were changed by addition of $\mathrm{NaOH}$ or $\mathrm{HCl}$ $(\mathrm{POCH})$ and were measured using an electronic pH-meter CP-401 (Hydromet) with electrode type ERH-13-6 at room temperature (RT). Urea spectroscopic purity was used (POCH). Refractive index and ORD were measured using a Pulfrich refractometer PR2 (Zeiss) and polarimeter Polamat A (Zeiss), respectively. Temperature dependence of optical rotation was measured at $366 \mathrm{~nm}$ using of $2 \mathrm{dm}$ jacketed cell. Faraday rotation measurements were performed with home-made spectropolarimeter in the spectral range $400 \div 600 \mathrm{~nm}$ [8]. Magnetic field at $1.09 \mathrm{~T}$ was produced by electromagnet and their intensity was monitored with teslameter RX-21b (Resonance Technology). Thermostat MK 70 (Zeiss) with a temperature control $\pm 0.1^{\circ} \mathrm{C}$ was used. The density value in the $288 \div 315 \mathrm{~K}$ temperature range were measured using a density meter type DMA 38 (Anton Paar). Specific optical rotation $[\alpha]_{\lambda}^{t}$ was calculated using standard relation $[\alpha]_{\lambda}^{t}=100 \alpha / c l$ where: $\alpha$ is in $\left[^{\circ}\right], c$ in $[\mathrm{g} / \mathrm{dL}]$ and $l$ in $[\mathrm{dm}]$. The Verdet constant was calculated using relation $V=\psi / B l$, where $\psi$ is in $\left[^{\circ}\right], B$ in [T] and $l$ in [m].

\section{Results and discussion}

The refractive index for several concentrations $(0.1 \div 10 \mathrm{~g} / \mathrm{dL})$ of dextran measured for both clinical dextran saline solution (for details see [7]) together with simultaneously measured density allow to calculate the mean optical polarizability from Lorentz-Lorenz formula [9]. The calculations were made with equivalent mol equal to glucose unit and $n$ approximated to $n_{\infty}$ by formula $\left(\lambda_{1}^{2} n_{1}-\lambda_{2}^{2} n_{2}\right) /\left(\lambda_{1}^{2}-\lambda_{2}^{2}\right)$ to avoid wavelength dependence of $\alpha$. The value of $\alpha$ as function of dextran concentration obtained in this way for Dex40 are given in Fig. 1b. For the $c=$ 

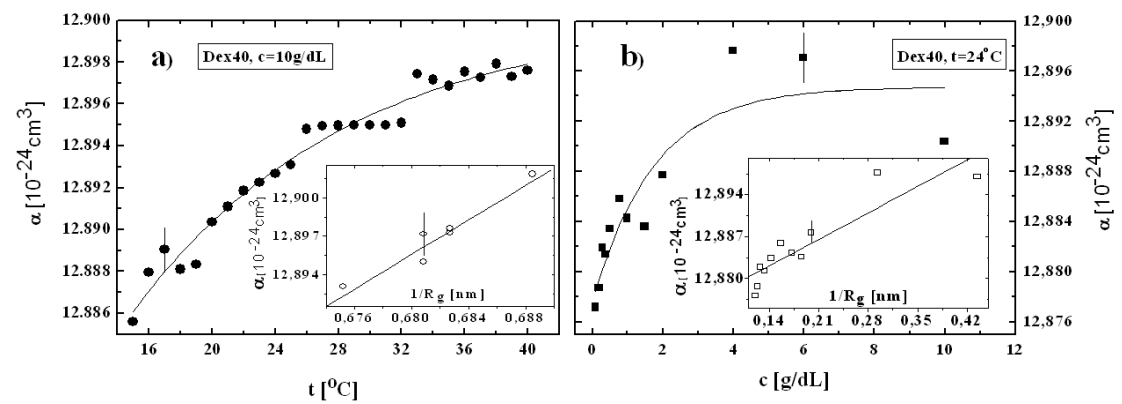

Fig. 1. The mean optical polarizability as a function of temperature (a) and dextran concentration (b) for dextran saline solution. In the insets the mean optical polarizability is shown as a function of reciprocal radius of gyration $-1 / R_{\mathrm{g}}$.

$10 \mathrm{~g} / \mathrm{dL}$, temperature dependence of $\alpha$ was also evaluated and is shown in Fig. 1a. In both cases optical polarizability tends to be a constant value as concentration and temperature increases. The value of $\alpha$ is slightly smaller from that of glucose, which was independently measured for aqueous glucose solution ( $c=10 \mathrm{~g} / \mathrm{dL}, \mathrm{RT})$.

Recently Hirata et al. [10] from small-angle X-ray spectroscopy (SAXS) experiments have provided information about molecular structure of moderately concentrated aqueous dextran solution $(0.125 \div 10 \mathrm{~g} / \mathrm{dL})$ for the dextran with the same $M_{\mathrm{w}}$ as in the present study. Using this data [10] the plot of $\alpha$ vs. $1 / R_{\mathrm{g}}$ is shown in the inset of Fig. 1b. Linear relation may be easily noticed. The temperature dependence of $\alpha$ vs. $1 / R_{\mathrm{g}}$ was also established using a set of data of $R_{\mathrm{g}}$ given by Güner [11] from viscosity measurements of aqueous dextran solution in the temperature range $25 \div 43^{\circ} \mathrm{C}\left(M_{\mathrm{w}}=40,000\right)$ (see inset of Fig. 1a). Although the range of temperature is small, still the linear $\alpha$ dependence from $1 / R_{\mathrm{g}}$ may be seen. It is worth to notice that similar to Güner data, $R_{\mathrm{g}}$ dependence as function of temperature may be obtained from the results of Nordmeier [12], in temperature $15 \div 60^{\circ} \mathrm{C}$, however for dextran with much higher molecular weight than in our study. Taking into account relations in the procedure for obtaining a radius of gyration $R_{\mathrm{g}}$ provided by Zimm and relation between Rayleigh ratio $R_{\Theta}$ and the mean optical polarizability [13], one may have established that: $\alpha \propto 1 / R_{\mathrm{g}}$. Therefore, the behaviour of $\alpha$ vs. $1 / R_{\mathrm{g}}$ depicted in Fig. 1 is in excellent agreement with theoretical prediction.

It was found that both dextrans are characterized by positive plain rotatory dispersion curves and obey the Drude equation [7]. The results of the $[\alpha]_{\lambda}^{24}$ dependence on the concentration of dextran in saline solution in the range $0.01 \div 10 \mathrm{~g} / \mathrm{dL}$ are presented in Fig. 2a. Two characteristic concentrations may be noticed, one $c_{1} \cong 0.02 \mathrm{~g} / \mathrm{dL}$ and second $c_{2} \cong 2 \mathrm{~g} / \mathrm{dL}$ (see Fig. $2 \mathrm{a}$ ).

Below $c_{1}$ the $[\alpha]_{\lambda}$ changes smoothly and linearly. Above this concentration it is not linear any more and changes randomly around constant value $[\alpha]_{366} \cong 570$ up to more or less to $c_{2} \cong 2 \mathrm{~g} / \mathrm{dL}$. When concentration is approaching $c_{2}$ the $[\alpha]_{\lambda}$ 

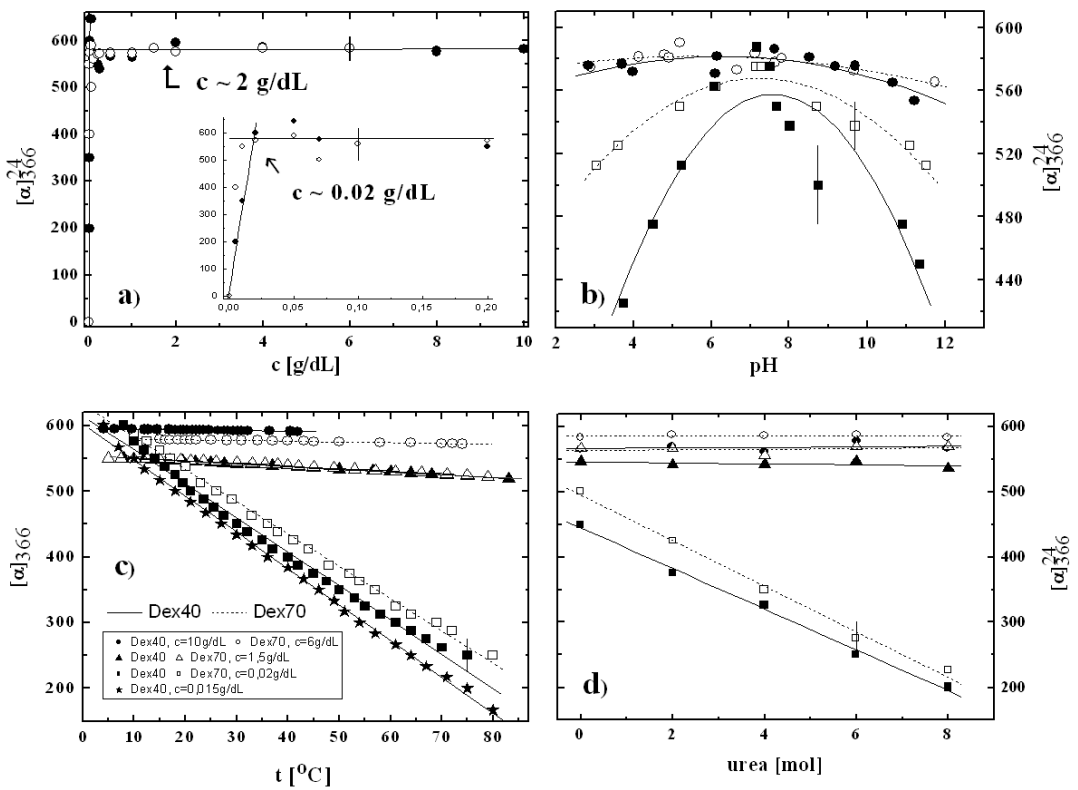

Fig. 2. The effect of the dextran concentration (a), pH environment (b), temperature (c), and urea (d) on the optical rotation of dextran saline solution. Full points and solid lines and empty points and dashed lines are for dextran with molecular weight $M_{\mathrm{w}}=40 \mathrm{kDa}$ and $M_{\mathrm{w}}=70 \mathrm{kDa}$, respectively.

is stable and constant up to $c=10 \mathrm{~g} / \mathrm{dL}$. Such behaviour was observed for several different batches and for dextran from both sources.

It is well known that dextran molecule forms a flexible polymer chain with glucose as repeating unit having a hydrogen-bonding donor and acceptor group. Therefore, in water, network chains between polymer segments or solvent can be made easily through hydrogen bonds or/and other intra- and/or intermolecular interactions. The changes in this network lead to conformation changes in dextran chain, which finally reflect in changing of measured specific optical rotation of dextran solution. This situation can be observed in Fig. 2, where specific optical rotation changes for respective concentration are shown under influence of temperature, $\mathrm{pH}$, and urea, the compound known as strongly breaking hydrogen bonds. The $[\alpha]_{\lambda}$ has quite different specific changes below and above these two characteristic concentrations. In diluted solution below $c_{1}$ when good condition for isolation of polymer chain appear, there are not any changes observed which may have character of the order-disorder transition. This is clearly seen for the $[\alpha]_{\lambda}$ vs. $t$ or urea concentration where no sigmoidal or jump-like, but rather monotonic smooth changes can be observed. More than $70 \%$ changes in the value of $[\alpha]_{\lambda}$ for $c=0.015 \mathrm{~g} / \mathrm{dL}$ (see Fig. 2c) can be related with the situation, where extended polymer coil will have contracted and shrunk in diameter and becoming more rod 
shaped when interaction between segments are breaking with raising temperature, $\mathrm{pH}$ or urea content. Above $c_{2}$ constant value of the $[\alpha]_{\lambda}$ with $c$ and very weak linear changes with $t$ and $\mathrm{pH}$ or urea content is observed. It means that very strong overlapping of chain segments occur and dextran chain appear in a coiled conformation as random or compact coil. In between $c_{1}$ and $c_{2}$ the $[\alpha]_{\lambda}$ changes suggest that strong intermolecular interaction between chain segments still exists and is not eliminated by dilution or other forces $(t, \mathrm{pH}$, urea). Branching can be a factor responsible for enhancement of interaction between segments. Although contribution of external forces in changes of the structure is increasing with decreasing solution concentration.

The $c_{2}$ value can be correlated with critical overlap concentration (coc) $c^{*}$ which is known for dextran and have been evaluated by different methods [14]. However, characteristic concentration $c_{1}$ was not observed and evaluated for dextran but tentatively it may be correlated with initial overlap concentration or so-called critical aggregation concentration (cac). Both concentrations show small molecular weight dependence as can be noticed in Fig. 2a. The cac value was evaluated for some hydrophobically modified dextrans $\left(M_{\mathrm{w}}=40,000\right)$ with different degree of substitution (DS) $[15,16]$. Approximation of the DS to zero gave the cac value for "pure dextran" in the range $0.014 \div 0.024 \mathrm{~g} / \mathrm{dL}$, in agreement with $c_{1}^{40}=0.016$ and $c_{1}^{70}=0.012 \mathrm{~g} / \mathrm{dL}$ obtained in present study. This corroborates assignment of $c_{1}$ as cac.

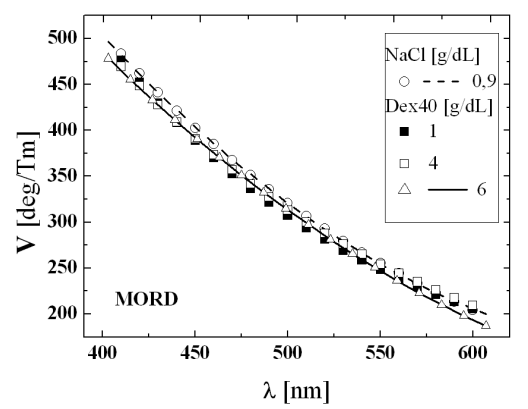

Fig. 3. The Verdet constant of dextran saline solution for several dextran concentrations and aqueous saline solution as a function of wavelengths.

Dispersion of the Faraday effect has been measured for several concentrations of dextran in the range of $c$ equal to $0.02 \div 10 \mathrm{~g} / \mathrm{dL}$ and for aqueous saline solution $(\mathrm{NaCl}-0.9 \mathrm{~g} / \mathrm{dL})$. The values of $V$ for dextrans Dex40 and Dex70 were found to be almost the same, so for clarity the results for Dex40 are only shown in Fig. 3. The Verdet constant is described by positive plain dispersion curve, which can be well described by equation similar to Drude one. The dispersion curve of dextran lies slightly lower than that one of saline solution and water (not shown in the figure). Faraday rotation $(\psi)$ very weakly depends on temperature as it may be 
expected for diamagnetic substances. In the $10 \div 85^{\circ} \mathrm{C}$ temperature range the value of $\psi$ changes less than $4 \%$ and nearly equally for dextran saline solution with $c$ equal to 10 and $0.02 \mathrm{~g} / \mathrm{dL}$. It means that such drastic changes of steric condition related to dextran chain conformation do not make any crucial contribution to magnetooptical rotation unless like it was observed for ORD.

\section{Conclusion}

The mean optical polarizability of dextran polymer was measured and it was found that changes of $\alpha$ are well described by relation $\alpha \propto 1 / R_{\mathrm{g}}$. Optical rotation of dextran allows to distinguish two characteristic concentrations which have been correlated to $c_{1}=$ cac and $c_{2}=c^{*}$. In diluted solution smooth changes of the $[\alpha]_{\lambda}$ do not indicate any order-disorder conformation transition in dextran chain in temperature $5 \div 85^{\circ} \mathrm{C}$. Faraday effect of dextran is smaller than for water and shows diamagnetic behaviour without any significant contribution related to polymer chain changes.

\section{Acknowledgments}

Authors would like to acknowledge Prof. P. Miecznik for the use of a density meter and Baxter Terpol Ltd. for dextran donation.

\section{References}

[1] A.N. de Belder, Dextran in: Industrial Gums, Eds. R.L. Whistler, J.N. BeMiller, Academic Press, New York 1993, p. 399.

[2] B.K. Denizli, H.K. Can, Z.M.O. Rzaev, A. Güner, Polymer 45, 6431 (2004).

[3] Q.A. Pankhurst, J. Connolly, S.K. Jones, J. Dobson, J. Phys. D, Appl. Phys. 36, R167 (2003).

[4] M.T. Telling, S.H. Kilcoyne, J. Phys., Condens. Matter 19, 026221 (2007).

[5] A.J. Stiepanovic, E.S. Stevens, K. Gekko, Macromolecules 13, 1471 (1981).

[6] K. Gekko, ACS Symposium Series 150, 415 (1981).

[7] M. Koralewski, F. Reinholz, J. Karoń, K. Waraczewski, Pol. J. Envir. Stud. 15, 66 (2006).

[8] M. Koralewski, Magnetochiraloptical Methods in Biology, Adam Mickiewicz University Press, Poznań 1991 (in Polish).

[9] M. Born, E. Wolf, Principles of Optics, Pergamon, Oxford 1984.

[10] Y. Hirata, Y. Sano, M. Aoki, H. Shohji, S. Katoh, J. Abe, S. Hitsukuri, H. Yamamoto, Carbohydrate Polymers 53, 331 (2003).

[11] A. Güner, J. Appl. Polym. Sci. 72, 871 (1999).

[12] E. Nordmeier, J. Phys. Chem. 97, 5770 (1993).

[13] S.F. Sun, Physical Chemistry of Macromolecules, Wiley, Hoboken New Jersey 2004.

[14] C.E. Ioan, T. Aberle, W. Burchard, Macromolecules 34, 326 (2001).

[15] M.R. Rodrigues, J. Carbohydr. Chem. 24, 733 (2005).

[16] N.A.B. Vieira, M.S. Moscardini, V.A. de Tiera, M.J. Tiera, Carbohydrate Polymers 53, 137 (2003). 